\title{
A headache with surprising outcome: first case of brucellosis caused by Brucella suis biovar 1 in Germany
}

\author{
Sabine Zange ${ }^{1,2} \cdot$ Kim Schneider $^{3} \cdot$ Enrico Georgi $^{1} \cdot$ Holger C. Scholz ${ }^{1,2} \cdot$ Markus H. Antwerpen ${ }^{1}$. Mathias C. Walter ${ }^{1}$. \\ Lothar Zoeller $^{1,2} \cdot$ Heiner von Buttlar ${ }^{1} \cdot$ Johannes P. Borde ${ }^{4,5}$
}

Received: 13 February 2019 / Accepted: 27 April 2019 / Published online: 9 May 2019

(C) The Author(s) 2019

\begin{abstract}
In July 2018, brucellosis was diagnosed in a German patient without a travel history to regions endemic for Brucella. Microbiological analysis, including whole-genome sequencing, revealed Brucella suis biovar 1 as the etiologic agent. Coregenome-based multilocus sequence-typing analysis placed the isolate in close proximity to strains originating from Argentina. Notably, despite a strong IgM response, the patient did not develop Brucella-specific IgG antibodies during infection. Here, we describe the clinical course of infection, the extensive epidemiological investigations, and discuss possible routes of transmission.
\end{abstract}

Keywords Brucella suis biovar $1 \cdot$ Whole-genome sequencing $\cdot$ Germany $\cdot$ Autochthonous $\cdot$ Serology

\section{Introduction}

Human brucellosis, caused by Brucella species, is a notifiable disease in Germany and belongs to the most common bacterial zoonotic diseases worldwide. Brucellosis is endemic in countries of the Mediterranean basin, Middle East, parts of Central and South America, Africa, and Asia. Symptoms occur within an incubation period of 10-21 days, in some cases up to several months. The clinical picture of human brucellosis is not specific, often accompanied

Electronic supplementary material The online version of this article (https://doi.org/10.1007/s15010-019-01312-7) contains supplementary material, which is available to authorized users.

Sabine Zange

sabinezange@bundeswehr.org

1 Bundeswehr Institute of Microbiology, 80937 Munich, Germany

2 National Consultant Laboratory for Brucellosis, 80937 Munich, Germany

3 Ortenauklinikum Offenburg-Gengenbach, Gastroenterology and Hepatology, 77654 Offenburg, Germany

4 Division of Infectious Diseases, Department of Medicine II, University of Freiburg, Medical Center and Faculty of Medicine, 79106 Freiburg, Germany

5 Praxis Dr. J. Borde/Gesundheitszentrum Oberkirch, 77704 Oberkirch, Germany with flu-like symptoms such as undulant fever, sweating, asthenia, myalgia, arthralgia, and headache. The agent can be translocated to almost any tissue or in vivo site, and in case of improper antibiotic treatment, the disease has a high risk of chronification and/or relapses. B. melitensis is the predominant species causing human brucellosis in most endemic regions, but $B$. abortus or $B$. suis might significantly contribute to the number of human infections in areas with extensive cattle or swine livestock farming, and therefore, the occurrence of the different Brucella species varies among geographic regions. Germany became officially free from brucellosis in cattle, sheep, and goats in 2002 and no autochthonous human infection has been reported ever since. However, annually, up to 50 human cases are imported to Germany from endemic regions. The majority of these cases (>95\%) are caused by B. melitensis, followed by B. abortus and $B$. suis, respectively. Importation occurs either through travellers or immigrants or via contaminated food products such as unpasteurized cheese or raw meat [1]. Despite being free of B. melitensis and B. abortus, wild boars and hares in Germany are known carriers of $B$. suis biovar 2, a biovar of $B$. suis which is of low human virulence, but may be transmitted to livestock, causing severe economic losses [2, 3]. In recent years, the isolation of $B$. suis biovars 1 and 3 from wild animals (wild boars) in Europe has been reported, but until now, no autochthonous human infection occurred [4]. Nevertheless, the virulence of biovars 1 and 3 
Table 1 Laboratory results at different timepoints

\begin{tabular}{|c|c|c|c|c|c|c|c|}
\hline Parameter (unit), (normal range) & 21.06 .2018 & 26.06 .2018 & 11.07.2018 & 20.07.2018 & 09.08.2018 & 24.08 .2018 & 23.10.2018 \\
\hline White cell count (per $\mathrm{mm}^{3}$ ) & 3760 & 2800 & 2200 & 3200 & 3210 & 2960 & 4300 \\
\hline Platelet count (per $\mathrm{mm}^{3}$ ) & 112,000 & 68,000 & 111,000 & 323,000 & 295,000 & 252,000 & 235,00 \\
\hline Hemoglobin (g/dl) & 15.6 & 13.0 & 9.8 & 11.7 & 12.0 & 13.6 & 15.7 \\
\hline Creatinine $(\mathrm{mg} / \mathrm{dl}),(0.6-1.1 \mathrm{mg} / \mathrm{dl})$ & 0.85 & 0.92 & 0.89 & 0.81 & 0.78 & 0.82 & 0.97 \\
\hline C-reactive protein $(\mathrm{mg} / \mathrm{dl}),(<0.5)$ & 6.3 & 5.8 & 4.4 & 0.5 & $<0.5$ & $<0.5$ & $<0.5$ \\
\hline Alanine aminotransferase $(\mathrm{U} / \mathrm{l}),(<45 \mathrm{U} / \mathrm{l})$ & 64 & 184 & 62 & 20 & 17 & 40 & 30 \\
\hline Aspartate aminotransferase $(\mathrm{U} / \mathrm{l}),(<45 \mathrm{U} / \mathrm{l})$ & 63 & 147 & 78 & 30 & 18 & 41 & 38 \\
\hline
\end{tabular}

towards humans is considered to be much higher, compared to biovar 2. Here, we describe the clinical course of the first B. suis biovar 1 infection in a German patient without a travel history to regions endemic for Brucella. An extensive epidemiological survey together with subsequent attempts to cultivate the agent from various potential sources as well as molecular typing were initiated to shed light on the possible route of infection.

\section{Case description}

A 56-year-old male patient presented in June 2018 to a family practice with a 3 -day history of headache, minimal photophobia, malaise, and febrile temperatures up to $38.4{ }^{\circ} \mathrm{C}$ (tympanic temperature). His medical history revealed arterial hypertension, hyperlipidemia, adiposity, and tobacco addiction. The patient reported no recent travel history, no animal contacts, or insect bites. He lives in the Ortenau district, a rural area in Southwestern Germany, is married and has three children. He is employed as an office worker in an industrial company producing metalwork. A follow-up visit the next day was scheduled on the basis of the preliminary diagnosis of a common cold. The initial blood samples showed a low platelet count of 112,000 per $\mathrm{mm}^{3}$, elevated liver function tests and a C-reactive protein at $6.3 \mathrm{mg} / \mathrm{dl}$ (normal $<0.5 \mathrm{mg} / \mathrm{dl}$ ) (Table 1). Serological screening for tick-borne encephalitis virus was negative. After a followup visit with persistent symptoms and febrile temperature, the patient was admitted to the neurological department of a local hospital. Advanced diagnostic workup, including blood cultures, cerebrospinal fluid (CSF) diagnostics regarding varicella zoster virus, herpes simplex virus (HSV), borreliosis, screening for viral hepatitis including Epstein-Barr virus and cytomegalovirus, human immunodeficiency virus, and Treponema pallidum, was done without any positive findings. There was a moderate CSF pleocytosis with 10 cells $/ \mu \mathrm{l}$ (Table 2). Abdominal sonography showed a marked hepatosplenomegaly and an infra-renal abdominal aortic aneurysm with $37 \mathrm{~mm}$ in diameter. The patient was discharged with the diagnosis of unspecific viral meningitis with coinciding hepatitis. This was in-line with cranial magnetic resonance
Table 2 CSF results

\begin{tabular}{ll}
\hline Parameter (unit), (normal range) & 25.06 .2018 \\
\hline White cell count (per/ $\mu \mathrm{l})$ & 10 \\
CSF protein $(\mathrm{mg} / \mathrm{dl}),(20-50)$ & 60 \\
Glucose $(\mathrm{mg} / \mathrm{dl})$ & 61 \\
Lactate $(\mathrm{mmol} / \mathrm{l}),(1.1-2.1)$ & 2.2 \\
\hline
\end{tabular}

CSF cerebrospinal fluid

imaging findings which indicated meningitis without signs of encephalitis and a small frontal white matter lesion.

One week later, the patient presented again to the primary care facility with the chief complaint of a laceration wound at the forehead caused by a transient loss of consciousness and fever up to $39.4^{\circ} \mathrm{C}$. The working diagnosis at that time was fever of unknown origin and the patient was admitted to the department of internal medicine. The initial five sets of blood cultures indicated the growth of Gram-negative rods. Sub-cultured isolates were initially identified as Brucella melitensis via matrix-assisted laser desorption ionization time-of-flight mass spectrometry (MALDI-TOF MS) by the external microbiological laboratory using the standard Biotyper® reference library and the security-relevant library for identification (Bruker Daltonik GmbH, Bremen, Germany). Anti-infective treatment was initiated with p.o. doxycycline (100 mg bid), p.o. rifampin ( $450 \mathrm{mg}$ bid), i.v. ceftriaxone ( $2 \mathrm{~g}$ bid), and i.v. gentamicin for 2 weeks, followed by oral outpatient antibiotic treatment with doxycycline $(100 \mathrm{mg}$ bid), rifampin (450 mg bid) for 4 weeks. Extended diagnostic workup with transesophageal echocardiography showed no masses or valvular defects. Total-body computed tomography with contrast material was performed showing subsegmental pulmonal embolism, hepatosplenomegaly, and the abdominal aortic aneurysm. To rule out osteoarticular infection, a total-body scintigraphy was performed, but there was no evidence of an osteoarticular focus. Symptoms improved on antimicrobial treatment and subsequent blood cultures showed no growth as expected. Four months later, at the end of October, laboratory results including white cell count, platelet count, and liver function tests were normalized 

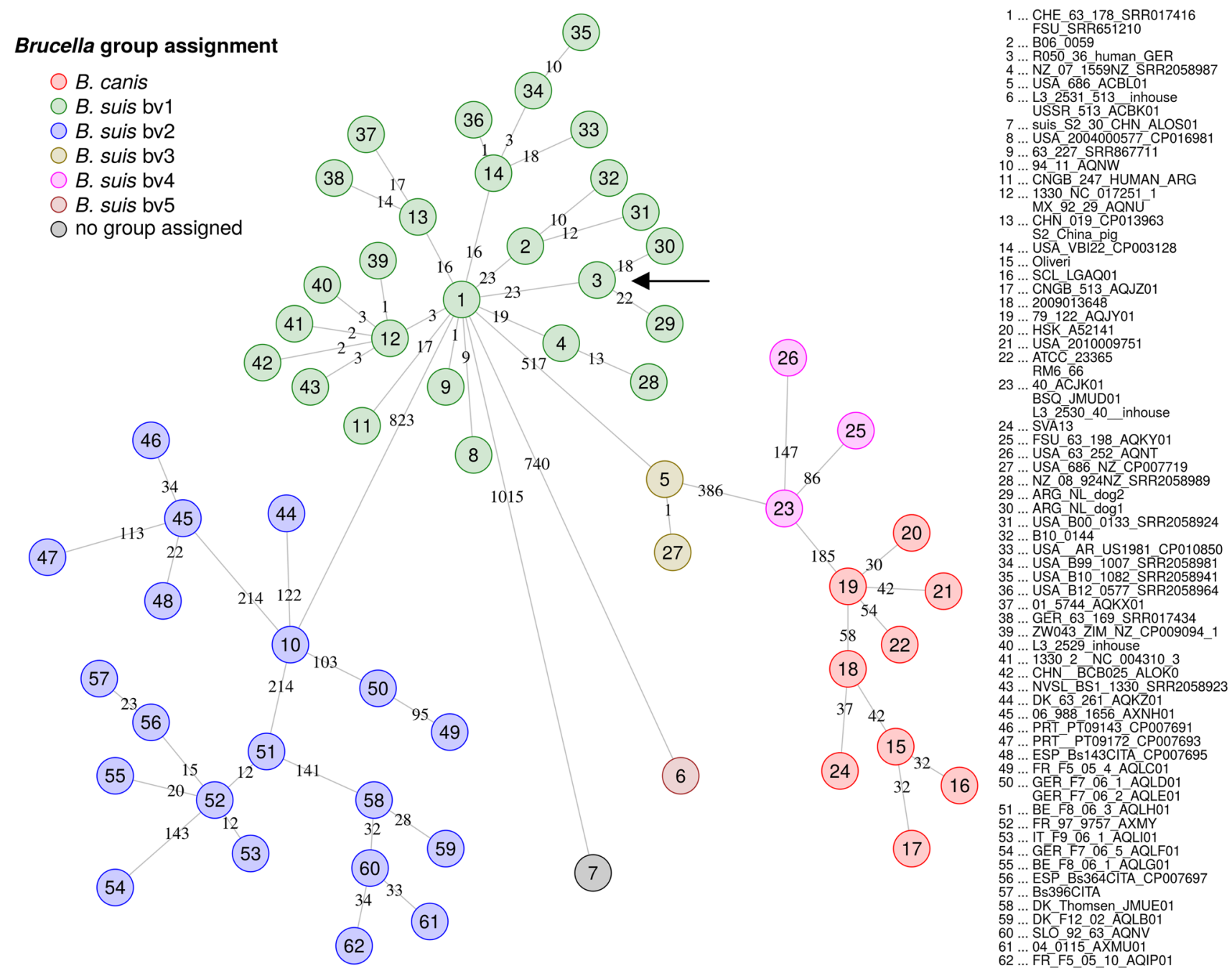

Fig. 1 Core-genome-based (cgMLST) minimum spanning tree of $70 \mathrm{~B}$. suis strains belonging to different biovars. The strain of this study is marked with an arrow. The tree was generated with SeqShere (Ridom GmbH, Muenster, Germany) using the cgMLST

scheme as described before [7]. This whole-genome shotgun project has been deposited at DDBJ/ENA/GenBank under the accession SRIH00000000. The version described in this paper is version SRIH01000000

(Table 1). Abdominal sonography control of the aortic aneurysm was pending.

\section{Advanced microbiology and epidemiological investigation}

The bacterial strain isolated from blood culture was transferred to the national consultant laboratory for brucellosis for confirmation of the species identification and antimicrobial susceptibility testing. Brucella was confirmed by polymerase chain reaction (PCR) targeting the genus-specific markers $I S 711$ and bcsp31. However, the species differentiating Brucella bruce-ladder PCR [5] identified the isolate as $B$. suis. The initial misidentification as $B$. melitensis by MALDI-TOF MS was due to a lack of $B$. suis reference spectra in the applied commercial database. To determine

the exact taxonomic affiliation, whole-genome sequencing on a MiSeq platform and subsequent in silico multilocus sequence typing (MLST), multiple-locus variable-number tandem-repeats analysis (MLVA-16), and single-nucleotide polymorphism (SNP) analysis using own and external scripts as described recently [6-8] were performed. The sequenced genome has been deposited at DDBJ/ENA/GenBank under the accession SRIH00000000. The genetic profile of the strain clearly revealed a member of the Brucella suis biovar 1 clade (Fig. 1) with closest proximity to strains from a dog fed with contaminated meat from Argentina. Antimicrobial susceptibility testing using broth microdilution showed sensitivity towards doxycycline, rifampin, trimethoprim/ sulfamethoxazole, gentamicin, and streptomycin. Unfortunately, no retained CSF sample was available for Brucella PCR testing. 
Table 3 Brucella serology results from different timepoints in parallel titration

\begin{tabular}{|c|c|c|c|}
\hline Parameter (unit), (reference) & 12.07.2018 & 13.08 .2018 & 24.08.2018 \\
\hline Brucella IgG antibodies $(\mathrm{U} / \mathrm{ml}),(<20)$ & 24 & 8 & 8 \\
\hline Brucella IgM antibodies $(\mathrm{U} / \mathrm{ml}),(<15)$ & 2368 & 2176 & 1536 \\
\hline Brucellacapt $^{\circledR}$ (titre),$(<1: 40)$ & $1: 80,960$ & $1: 80,960$ & $1: 40,480$ \\
\hline
\end{tabular}

IgG immunoglobulin $\mathrm{G}$; $I g M$ immunoglobulin $\mathrm{M}, U$ units
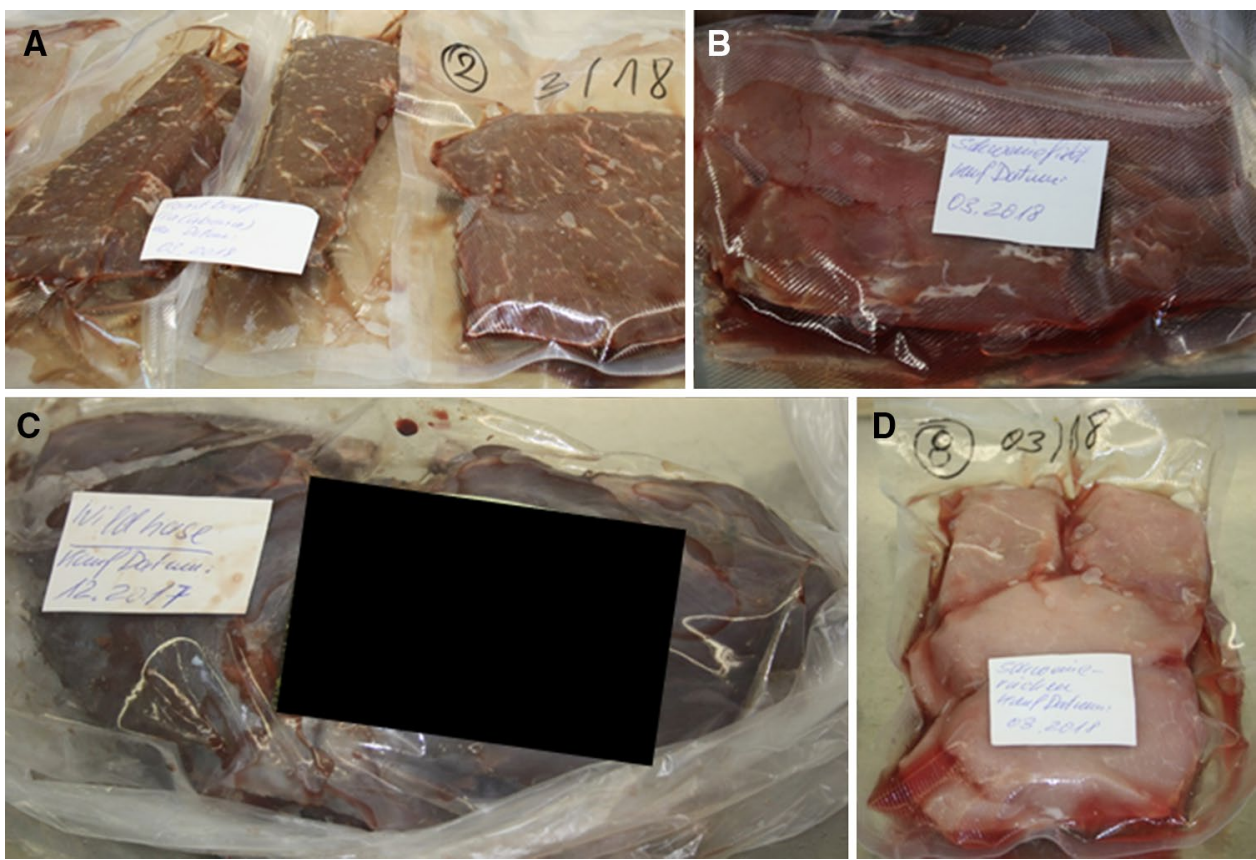

Fig. 2 Content of the patients freezer. a Beef steak (origin unknown), $\mathbf{b}$ fillet of pork (origin unknown), $\mathbf{c}$ leg of hare from Argentina, $\mathbf{d}$ saddle of pork (origin unknown)
Brucella serology using Brucella $\operatorname{IgM}$ and IgG antibody enzyme-linked immunosorbent assay (ELISA) (Virion/ Serion, Wuerzburg, Germany) and Brucellacapt ${ }^{\circledR}$ (Vircell, Granada, Spain) resulted in highly positive IgM antibody and Brucellacapt ${ }^{\circledR}$ values, but unexpectedly revealed only borderline IgG antibody values. The follow-up sera showed also no IgG class-switch of Brucella-specific antibodies even two months later, while IgM values were slightly decreasing (Table 3).

The patient reported no recent travel history, no consumption of (unpasteurized) dairy products, no animal contacts, hunting experiences, and no insect bites. He lives in rural Southern Germany, and in the weeks before onset of symptoms, he did a lot of gardening at his property, where he often observed wild hares.

Extensive interviewing of the patient revealed excessive meat consumption. He is in the habit of buying batches of meat from a wholesale market and to process the meat in his garage. He cut his finger during preparation of minced meat (consisting of pork and beef) approximately two months before the onset of symptoms. To elucidate the mode of transmission, the patient and his family were visited at home. His freezer contained the leg of a hare from
Argentina, saddle of pork (origin unknown), fillet of pork (origin unknown), and beef steak from Botswana, but unfortunately, no retained sample of the minced meat mentioned above (Fig. 2). From a previously consumed second leg of a hare from Argentina, also no retained sample was available. He denied the consumption of meat from wild boar.

All available meat samples showed negative results in Brucella PCR testing (targets: bcsp31, IS711) and all microbiological cultures were negative for Brucella species. The family, including his wife and the two sons of the patient, showed no symptoms and their Brucella IgM and IgG antibody ELISA tests were negative one month after diagnosis in our patient. The third child was not living in the same location and, therefore, was not tested.

\section{Discussion}

B. suis is frequently isolated in regions of South America mainly in Argentina, the USA, and European countries [9]. The species is subdivided into five biovars. In Europe, B. suis biovar 2 is responsible for infections of wild boars and hares $[2,10]$, whereas $B$. suis biovar 1 is the most frequently found 
biovar in South America isolated from humans and pigs [9]. The usual route of transmission to humans is the ingestion of contaminated food, e.g., unpasteurized dairy products or undercooked meat from infected animals. The disease may also be acquired by handling infected animals or inhalation of infectious aerosols. In particular, B. suis biovar 1 transmissions were described from Argentina in slaughterhouse workers. Affected employees were infected by injuries when processing pork meat, contaminated aerosols, conjunctival splashes, or direct bacterial entry through skin lesions [11, 12]. From other countries (The United States, Australia, Turkey), B. suis biovar 1 infections have been described in humans who hunt and handle feral pigs and are associated with dressing the killed animals or processing their meat [13-15]. Furthermore, in a dog from The Netherlands suffering from a $B$. suis biovar 1 infection, the risk of infection was determined to be a raw meat-based diet. In this case, the contaminated meat consisted of carcasses of hares from Argentina [16]. To trace back such importations, molecular epidemiological analyses using modern molecular fingerprinting techniques such as MLVA or genome-wide mutation analysis are crucial. Unfortunately, in our case, the source of infection could finally not be elucidated by means of bacterial culture of retained meat samples nor by molecular fingerprinting. All investigated meat samples were Brucella negative. No retained sample exists from the minced meat (which was most likely the source of infection). Therefore, evidence-based source attribution was not possible. Due to the limited number of $B$. suis biovar 1 genome sequences in the databases and sparse or possibly even erroneous metadata on geographical origin of the strains, a spatial attribution of the isolate was also not possible. The closest genetic relatives with 18 and 23 SNPs, respectively, originate from Argentina. However, because of limited genomic data in the databases, this epidemiological link remains speculative. The route of transmission also remains a matter of speculation, but occurred most likely during preparation of minced meat when the patient cut his finger. Since the origin of the processed meat is unknown and $B$. suis biovar 1 is distributed globally, the pork meat is a possible source of infection. Despite being tested negative for $B$. suis in the remaining batch, the leg of a hare from Argentina can also not be entirely ruled out as a potential source of infection, as the closest genetic relatives originate from Argentina. Infection via ingestion seems less likely, because household contacts sharing the same food preferences showed no symptoms and were serologically negative regarding Brucella species.

An interesting and unexplained feature of the presented clinical course is the missing $\operatorname{IgG}$ antibody response, despite the presence of a high level of Brucella-specific IgM antibodies. Extended immunological workup showed no evidence of immune deficiency. Immune responses to vaccine antigens such as measles virus or frequent pathogens such as HSV showed appropriate IgG levels. However, published case reports indicate that in rare cases of $B$. suis infections, a general immune response or $\mathrm{IgG}$ seroconversion is missing [17]. Up to date, it remains a matter of debate, which host and/or pathogen factors might be relevant in this context. Analysis of the $w b k$ genetic island encoding for lipopolysaccharide synthesis revealed no peculiarities which might have explained the lack of specific IgG antibody response (data not shown).

In conclusion, the detection of $B$. suis biovar 1 in a patient without a travel history to regions endemic for Brucella could be due to an autochthonous infection acquired from wild animals, but also due to the import of contaminated food. Although the hypothesis of an infection via imported meat seems to be more likely in our case, this should alert physicians of a zoonotic disease acquirable also in Western Europe. Since $B$. suis biovar 1 is highly pathogenic to humans when compared to biovar 2, the occurrence of biovar 1 in Western Europe is of great importance. Therefore, brucellosis should be considered in all patients with unexplained febrile illness even without travel history to endemic regions, particularly when they have contact to wild animals or relevant food preferences.

Acknowledgements The Bundeswehr Institute of Microbiology participates in the Joint Action EMERGE funded by the European Commission (CHAFEA no. 677 066). We gratefully acknowledge Hendrik Jan Roest (Wageningen Bioveterinary Research, Lelystad, The Netherlands) for providing unpublished WGS data of some B. suis biovar 1 strains.

Author contributions SZ wrote the manuscript and performed serological and molecular biological diagnostic assays. KS managed the patient during his hospital stay. JB managed the patient at the outpatient clinic, collected the detailed medical history, and contributed in drafting the manuscript. EG performed the in silico MLST and MLVA analyses, plotted the data, and edited the manuscript. HCS executed a thorough SNP analysis of the isolate. MCW developed and optimized the NGS workflow. MHA supervised and reviewed the NGS sequencing and analyses. LZ supervised the diagnostic processes. HvB was involved in the testing of meat samples and the immunological investigations. All authors reviewed and approved the final version of the manuscript.

Funding No external funding. All investigations were funded by own contribution.

\section{Compliance with ethical standards}

Conflict of interest All authors do not declare any conflict of interest.

Ethical approval This article does not contain any studies with human or animal subjects.

Informed consent Informed consent was obtained from the patient described in the case report section. 
Open Access This article is distributed under the terms of the Creative Commons Attribution 4.0 International License (http://creativeco mmons.org/licenses/by/4.0/), which permits unrestricted use, distribution, and reproduction in any medium, provided you give appropriate credit to the original author(s) and the source, provide a link to the Creative Commons license, and indicate if changes were made.

\section{References}

1. Norman FF, Monge-Maillo B, Chamorro-Tojeiro S, Pérez-Molina JA, López-Vélez R. Imported brucellosis: a case series and literature review. Travel Med Infect Dis. 2016;14:182-99. https://doi. org/10.1016/j.tmaid.2016.05.005.

2. Melzer F, Lohse R, Nieper H, Liebert M, Sachse K. A serological study on brucellosis in wild boars in Germany. Eur J Wildl Res. 2007;53:153-7.

3. Kreizinger Z, Foster JT, Rónai Z, Sulyok KM, Wehmann E, Jánosi $\mathrm{S}$, Gyuranecz M. Genetic relatedness of Brucella suis biovar 2 isolates from hares, wild boars and domestic pigs. Vet Microbiol. 2014;172:492-8. https://doi.org/10.1016/j.vetmic.2014.05.031.

4. Cvetnic Z, Spicic S, Jukic B, et al. Brucella suis infection in domestic pigs and wild boar in Croatia. Rev Sci Tech. 2009;28:1057-67.

5. García-Yoldi D, Marín CM, de Miguel MJ, Muñoz PM, Vizmanos JL, López-Goñi I. Multiplex PCR assay for the identification and differentiation of all Brucella species and the vaccine strains Brucella abortus S19 and RB51 and Brucella melitensis Rev1. Clin Chem. 2006;52:779-81. https://doi.org/10.1373/clinc hem.2005.062596.

6. Georgi E, Walter MC, Pfalzgraf MT, Northoff BH, Holdt LM, Scholz HC, Zoeller L, Zange S, Antwerpen MH. Whole genome sequencing of Brucella melitensis isolated from 57 patients in Germany reveals high diversity in strains from Middle East. PLoS One. 2017;12:e0175425. https://doi.org/10.1371/journ al.pone. 0175425 .

7. Janowicz A, De Massis F, Ancora M, Cammà C, Patavino C, Battisti A, Prior K, Harmsen D, Scholz H, Zilli K, Sacchini L, Di Giannatale E, Garofolo G. Core genome multilocus sequence typing and single nucleotide polymorphism analysis in the epidemiology of Brucella melitensis infections. J Clin Microbiol. 2018;56:e00517-8. https://doi.org/10.1128/JCM.00517-18.

8. Vergnaud G, Hauck Y, Christiany D, Daoud B, Pourcel C, Jacques I, Cloeckaert A, Zygmunt MS. Genotypic expansion within the population structure of classical Brucella species revealed by MLVA16 typing of 1404 Brucella isolates from different animal and geographic origins, 1974-2006. Front Microbiol. 2018;12:1545. https://doi.org/10.3389/fmicb.2018.01545.

9. Lucero NE, Ayala SM, Escobar GI, Jacob NR. Brucella isolated in humans and animals in Latin America from 1968 to 2006. Epidemiol Infect. 2008;136:496-503.

10. Mailles A, Ogielska M, Kemiche F, Garin-Bastuji B, Brieu N, Burnusus Z, Creuwels A, Danjean MP, Guiet P, Nasser V, Tourrand B, Valour F, Maurin M, O'Callaghan D, Mick V, Vaillant V, Jay M, Lavigne JP, DE Valk H. Brucella suis biovar 2 infection in humans in France: emerging infection or better recognition? Epidemiol Infect. 2017;145:2711-6. https://doi.org/10.1017/S0950 268817001704.

11. Wallach JC, García JL, Cardinali PS, Seijo AP, Benchetrit AG, Echazarreta SE, Garro SL, Deodato B, Baldi PC. High incidence of respiratory involvement in a cluster of Brucella suis-infected workers from a pork processing plant in Argentina. Zoonoses Public Health. 2017;64:550-3. https://doi.org/10.1111/zph.12339.

12. Escobar GI, Jacob NR, López G, Ayala SM, Whatmore AM, Lucero NE. Human brucellosis at a pig slaughterhouse. Comp Immunol Microbiol Infect Dis. 2013;36:575-80. https://doi. org/10.1016/j.cimid.2013.06.001.

13. Kutlu M, Cevahir N, Erdenliğ-Gürbilek S, Akalın Ş, Uçar M, Sayın-Kutlu S. The first report of Brucella suis biovar 1 isolation in human in Turkey. J Infect Public Health. 2016;9:675-8. https ://doi.org/10.1016/j.jiph.2016.01.011.

14. Carrington M, Choe U, Ubillos S, Stanek D, Campbell M, Wansbrough L, Lee P, Churchwell G, Rosas K, Zaki SR, Drew C, Paddock CD, Deleon-Carnes M, Guerra M, Hoffmaster AR, Tiller RV, De BK. Fatal case of brucellosis misdiagnosed in early stages of Brucella suis infection in a 46-year-old patient with Marfan syndrome. J Clin Microbiol. 2012;50:2173-5. https://doi. org/10.1128/JCM.00573-12.

15. Starnes CT, Talwani R, Horvath JA, Duffus WA, Bryan CS. Brucellosis in two hunt club members in South Carolina. J S C Med Assoc. 2004;100:113-5.

16. van Dijk MAM, Engelsma MY, Visser VXN, Spierenburg MAH, Holtslag ME, Willemsen PTJ, Wagenaar JA, Broens EM, Roest HIJ. Brucella suis infection in dog fed raw meat, the Netherlands. Emerg Infect Dis. 2018;24:1127-9. https://doi.org/10.3201/eid24 06.171887.

17. Naha K, Dasari S, Pandit V, Seshadri S. A rare case of seronegative culture-proven infection with Brucella suis. Australas Med J. 2012;5:340-3. https://doi.org/10.4066/AMJ.2012.1177. 